\title{
Void formation during film growth: A molecular dynamics simulation study
}

\author{
Richard W. Smith ${ }^{\mathrm{a})}$ and David J. Srolovitz \\ Department of Materials Science and Engineering, The University of Michigan, Ann Arbor, \\ Michigan 48109-2136
}

(Received 21 September 1995; accepted for publication 19 October 1995)

\begin{abstract}
Two-dimensional, nonequilibrium molecular dynamics simulations have been applied to study the structure of thin films grown on single-crystal Lennard-Jones substrates. The principal microstructural features to develop within these films are single vacancies and small voids which tend to be slightly elongated and to be aligned in the growth direction. Both the void volume and the mean surface roughness of the films are found to be decreasing functions of substrate temperature and deposition kinetic energy. Voids are shown to form as a consequence of both surface roughness and shadowing effects. The attraction between deposited atoms and the sides of surface depressions lead to the formation of outgrowths from the sidewalls of the surface depression. These outgrowths shadow the open void beneath them and continue to grow across the voids by interaction with the depositing atoms until a continuous bridge is formed that closes off the void. Since this bridging mechanism leaves behind a surface depression above the closed-off void, new voids tend to form above it. This leads to the alignment of voids along the film growth direction. The spacing of the resultant void tracks is correlated with the wavelength of the surface roughness. Increasing temperature and deposition kinetic energy enhancing surface mobility leads to an increase in the wavelength of the surface roughness and hence an increase in the spacing between void tracks. Edge dislocations tend to form within voids as a natural consequence of the void bridging process, however nondislocated voids are also observed. (c) 1996 American Institute of Physics. [S0021-8979(96)03303-2]
\end{abstract}

\section{INTRODUCTION}

The mechanical and electrical properties of physical vapor deposited thin films are controlled, in large part, by the microstructure, the state of stress within the film, and dislocations at the film/substrate interface. The nature of the microstructure and the magnitude of the stresses within the film are known to be sensitive to the conditions under which the film was deposited. For example, films grown at very low temperature tend to be porous (zone I microstructures), those grown at intermediate temperature tend to have a columnar grain structure and little porosity (zone II microstructures), and those grown at elevated temperature have either a very coarse or three-dimensional grain structure (zone III microstructures). ${ }^{1,2}$ Similarly, bombardment of the growing film with energetic species can lead to compressive stresses within the film, while film growth in the absence of bombardment commonly leads to the formation of tensile stresses. Therefore, control of film microstructure, stress and, hence, properties must be predicated upon the judicious manipulation of deposition conditions. The present study focuses on the development of voids within the film during film growth. In particular, we employ the molecular dynamics (MD) simulation method to examine the effects of variation of temperature and the kinetic energy of the depositing species on void formation. Our goal is the clear elucidation of void formation mechanisms and the factors that influence them.

Several authors ${ }^{3-17}$ have demonstrated the value of applying detailed atomistic computer simulations to the study

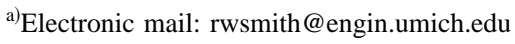

of thin film deposition. Simulations based on Monte Carlo (MC), MD, and ballistic deposition have all been applied for this purpose. Ballistic deposition simulations typically track atoms emitted from a source along a straight line trajectory to the point where they strike a target. The atoms are assumed to either reflect from the surface, stick where they hit, or locally relax into the cradle site closest to the point of impact. ${ }^{3,4}$ This approach typically neglects the dynamics and transport of the deposited atom once it impinges upon the surface. Liu et al. ${ }^{3}$ have used the ballistic deposition treatment in the SIMBAD program to study several basic aspects of microstructural formation (e.g., grain size, orientation) of films grown over topographical features such as grooves in thin film metallization in integrated circuits.

Kinetic MC simulations employ calculations of the rates of various types of atomistic events involving the atoms at the surface and a MC algorithm to determine which events actually occur in any given time interval. This approach can provide a more realistic, statistically valid picture of the evolving surface that includes such effects as diffusion, surface step migration, desorption, etc. Activation energies for diffusion and other processes must be estimated from external sources (experiment or other types of simulations). Applications using this method often select initial sites for atom deposition in much the same way as done in ballistic deposition studies, but once the atoms are attached to the surface (in perfect lattice sites), they are allowed to diffuse. Individual atomic jumps are attempted at random and succeed in accordance with a Boltzmann probability distribution, $P=\exp (-E / k T)$, where the migration energy for the jump is estimated from the number and spatial arrangement of neighbors surrounding the diffusing atom. Kinetic MC simulations 
have been employed to study surface roughening due to the nucleation and growth of islands, ${ }^{5-8}$ the deposition rate dependence of the transition temperature between columnar and densely packed microstructures, ${ }^{9}$ film growth on quasicrystals, ${ }^{10}$ the formation of columns during twocomponent deposition, ${ }^{11}$ etc.

In MD simulations of film growth, the complete trajectories of each atom in the system are calculated in a deterministic manner by integrating Newton's equation of motion forward in time. The trajectory of each atom is determined by the instantaneous forces on it due to its interaction with all of the other atoms in the system and all external fields. Therefore, this approach properly accounts for the full dynamics of thin film deposition. This is in contradistinction to MC methods, which only represent the dynamics in a statistical sense and do not account for important collective (multiatom) dynamic effects. All requisite information is computed directly from the spatial arrangement of the atoms and the interatomic potentials. The method is, however, limited in the length of time over which processes may be simulated because of the significant computational burden associated with employing an appropriately small time step (usually of the order of $10^{-2}$ times the vibrational period); typical calculations span only a few nanoseconds. MD calculations have been used to study accommodation and diffusion of adatoms on flat and vicinal film surfaces, ${ }^{12}$ formation and relaxation of columns in two-dimensional films, ${ }^{13,14}$ development of average surface stresses due to high energy atom and ion bombardment, ${ }^{15}$ structure and segregation in twocomponent films, ${ }^{16}$ the formation of amorphous films atop crystalline $\mathrm{Si}$ substrates, ${ }^{17}$ etc.

The purpose of the present work is to understand the factors affecting void formation during film growth. Toward this end, the present article describes the results of an atomistic MD simulation study. Since the purpose of this study is to understand void formation generically, the interatomic potentials employed are of the simple Lennard-Jones type. Film growth simulations were performed over a range of substrate temperatures and the kinetic energy of the deposition flux. The results, presented below, help identify the basic mechanism of void formation, the influence of temperature and kinetic energy on void size and density, and the tendency of voids to form in vertical tracks. ${ }^{18-20}$

\section{METHODS}

The simulation technique employed in this work is the now standard MD method, which was developed for performing atomistic simulations in the late $1950 \mathrm{~s} .^{21}$ In this method, the trajectories of each atom in a computational cell are followed through time by integrating Newton's classical equations of motion. The integration is performed in a stepwise manner over finite time steps using a fifth-order, Nordsiek, predictor-corrector method. ${ }^{22}$ The forces driving the motion of the atoms are computed from the gradient of the potential energy of the system with respect to the positions of each particle. In this work, we express the potential energy of the system as a sum over all pairs of atoms of a pairwise interaction energy given by the familiar Lennard-Jones potential,

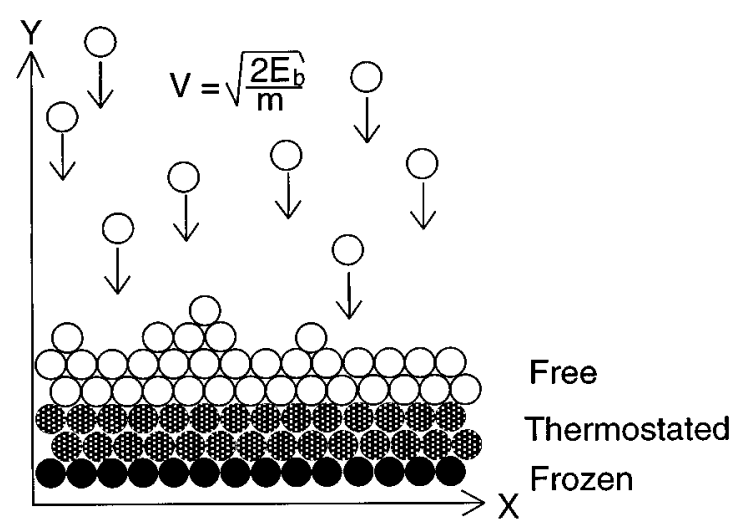

FIG. 1. Computational cell depicting initial substrate lattice and atoms impinging onto the surface. The bottom layer of the substrate is frozen, the two layers above it are thermostated, and atoms in subsequent layers are free of external forces. The range of the thermostated region increases with film thickness.

$$
U\left(r_{i j}\right)=\epsilon\left[\left(\frac{r_{0}}{r_{i j}}\right)^{12}-2\left(\frac{r_{0}}{r_{i j}}\right)^{6}\right]
$$

where $U\left(r_{i j}\right)$ is the interaction energy between atoms $i$ and $j$ separated by distance $r_{i j}, \epsilon$ is the strength of the interaction, and $r_{0}$ is a characteristic length scale. Although Eq. (1) extends over all atom pairs, for computational simplicity the potential is usually truncated at a finite distance, beyond which the interaction is taken to be zero. We have chosen our cutoff distance $r_{c}$ to be $2.1 r_{0}$, and have multiplied Eq. (1) between $r_{0}$ and cutoff by a cubic polynomial chosen such that both the modified $U(r)$ and its first derivative with respect to $r$ go smoothly to zero as $r$ approaches the cutoff distance. These conditions are required to avoid discontinuities in energy and force at the cutoff. Although the interaction potential has contributions from first, second, and third nearest neighbors in the perfect crystal, the contribution of the second and third neighbors to the total energy are minimal. Hence, $r_{0}$ may be regarded as the equilibrium, nearestneighbor atomic separation and $\epsilon$ as half the bond energy at that separation.

The computational cell is two dimensional and lies in the $X Y$ plane. Periodic boundary conditions are employed along the $X$ direction (i.e., along the substrate surface) and open boundary conditions are employed in the $Y$ direction (i.e., normal to the substrate surface). Substrate atoms are initially arranged on a triangular lattice and atoms comprising the deposition flux impinge from above, along the negative $Y$ direction (see Fig. 1). In the simulations discussed below, the substrate consists of three atomic layers. The atoms on the bottom layer of the substrate are frozen in space to prevent the substrate from being distorted or dissociated by the incident atoms and to prevent the entire system from moving through space due to the momentum absorbed from the deposition flux. Atoms on the top two layers of the substrate are "thermostated" in order to hold the substrate at the desired temperature. The velocities of the atoms on these layers are initially randomly chosen from a two-dimensional Maxwellian distribution corresponding to the desired temperature, and then fictitious external forces are applied through- 
out the duration of the simulation to maintain the average temperature of these atoms at the desired level. ${ }^{23}$

As the film grows, it is necessary to increase the height of the thermostated region to prevent excessive heating of the film. The kinetic energy of the atoms from the deposition flux and the sublimation energy liberated as these atoms condense onto the crystal act as a surface heat source. In a metallic crystal, heat is conducted through the film to the substrate and radiated from the surface. Both of these effects are electronic in origin and hence are not present in these simulations. In deposition of metallic films, the temperature of the surface does not typically greatly exceed that of the substrate. In the present Lennard-Jones model simulations, heat can be removed only by lattice vibrations which constitute only a small fraction of the thermal conductivity in the actual metallic solid. To compensate for this deficiency in this model, fictitious external forces are used to extract heat at a rate more reasonable for metallic films and thus provide nearly constant temperature. In this study, all atoms lying more than ten equilibrium lattice spacings beneath the lowest point on the film surface are thermostated, as described above for the top layers of the substrate. As the film grows, the top of the thermostated region moves with it. This does not greatly affect the dynamics since atoms in the thermostated region still respond to forces from the atoms in the nonthermostated region and each other. Furthermore, as shown below, most of the film growth dynamics occurs within one or two atomic spacings of the surface.

The atoms in the incident beam are all deposited along the negative $Y$ direction (i.e., a collimated flux) with a constant velocity dictated by the desired deposition flux energy $\left(E_{b}=m V^{2} / 2\right)$. The atoms are introduced at uniform time intervals at a constant height above the film at random positions in $X$. Owing to the short times accessible with MD, the present simulations are performed using a very high deposition flux. In order to diminish the effects of this high deposition rate on the dynamics of the atoms when they impinge upon the substrate and to avoid unphysical gas phase dynamics, the deposition flux is manipulated to ensure that the depositing atoms do not interact with each other prior to impingement on the surface. This is achieved by assuring that new atoms are introduced a distance greater than the interaction cutoff distance from any other atoms in the deposition flux. This condition may be achieved simply by limiting the deposition rate to rates such that a new atom is not introduced until the previous atom has fallen a distance equal to the interaction cutoff distance $r_{c}$. This procedure prevents interactions between the atoms even in the extreme case of two subsequent particles being deposited at the same $X$ location. Under these conditions, the minimum allowed time between deposition events is $\tau_{d}=r_{c} / V$, where $V$ is the velocity of the deposited atoms in the $-Y$ direction. This minimum time may be shortened by restricting the range of $X$ positions at which a new atom may be introduced to those values where the atom will not lie within the interaction distance of any of the previous atoms. Selection of the $X$ position of each new atom is made at random from within this restricted range.

If the atoms in the deposition flux have sufficiently large kinetic energy, they may reflect off of the surface or eject other surface atoms back up into the vapor. These reflected or ejected atoms can interact with depositing atoms and destroy the collimation and monoenergetic nature of the flux (leading to atomic clustering in the vapor). This becomes increasingly likely as the kinetic energy of the depositing atoms increases or the binding of an atom to the surface decreases (e.g., large roughness or high temperature). In order to prevent this phenomena from occurring, the atoms in the vapor above the film are searched for atoms with velocities differing from that at which the atoms are initially introduced. All such atoms are removed from the vapor.

Once the simulation is initialized, particle positions are recorded as a function of time and used to make MD "movies" of the growing films. In addition to the positions, local stress tensors are also recorded for each atom in the system. The stress, in dynamic simulations, is comprised of a static term reflecting the interatomic forces between particles and a kinetic term which depends on the velocity of the particles, ${ }^{24}$

$$
\sigma_{i}^{\alpha \beta}=-\frac{1}{\Omega_{i}}\left(\frac{1}{2} \sum_{j \neq i} F_{i j}^{\alpha} r_{i j}^{\beta}+M_{i} V_{i}^{\alpha} V_{i}^{\beta}\right),
$$

where, $\sigma_{i}^{\alpha \beta}$ is the stress tensor at the position of the $i$ th atom, $\Omega_{i}$ is the atomic volume of the $i$ th atom, $F_{i j}^{\alpha}$ is the $\alpha$ th component of the force on atom $i$ due to atom $j, r_{i j}^{\beta}$ is the $\beta$ th component of the vector distance from atom $i$ to atom $j, M_{i}$ is the mass of the $i$ th atom, and $V_{i}^{\alpha}$ is the $\alpha$ th component of the velocity of the $i$ th atom. The surface roughness of the film $R$ is also measured at several times during the deposition. $R$ is defined here, conveniently although unconventionally, as the total length of the actual surface minus the width of the film in the $X$ direction and then normalized by dividing by the width of the film. The arc length is measured in a piecewise linear fashion with linear segments between neighboring surface atoms. A perfectly smooth surface, therefore, corresponds to $R=0$.

In the present simulations, the physical parameters $\left(r_{0}, \epsilon\right.$ and $M$ ) were all set to unity. The velocity of the deposited atoms is $V=\sqrt{2 E_{b} / M}$ and the basic unit of time $\tau$ $=\sqrt{M r_{0}^{2} / \epsilon}$. The time step used in the integration of Newton's equation of motion was variable and was determined in terms of the maximum velocity at each time step $\Delta t=r_{0} /\left(200 V_{\max }\right)$. In order to give a physical feel for these parameters in terms of a real material, we have estimated these values for Ni. For $\mathrm{Ni}$ we find: $r_{0}=0.249 \mathrm{~nm}, \epsilon=0.74$ $\mathrm{eV}, M=9.7 \times 10^{-26} \mathrm{~kg}$, and $\tau=1.6 \times 10^{-13} \mathrm{~s}$. This yields a surface energy of the order of $2 \mathrm{~J} / \mathrm{m}^{2}$. While all of the results reported below are in terms of the fundamental parameters $r_{0}, \epsilon, M$, and $E_{b}$, the data can be converted to more physically meaningful numbers using the values for $\mathrm{Ni}$ quoted here.

\section{RESULTS}

Microstructures of films grown at substrate temperatures of $T=0 \epsilon / k_{B}, 0.0625 \epsilon / k_{B}, 0.1250 \epsilon / k_{B}, 0.1875 \epsilon / k_{B}$, and $0.2500 \epsilon / k_{B}$ with a deposition kinetic energy of $E_{b}=0.80$ $\epsilon$ /atom are shown in Fig. 2. The predominant microstructural features in these films are voids and vacancies. Both the 
(a) $T=0.0 \varepsilon / k$

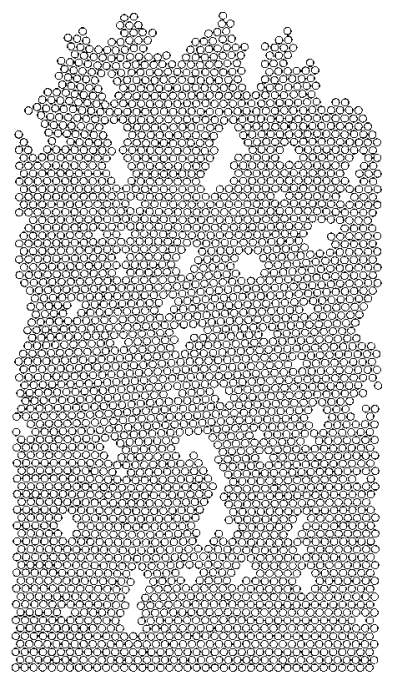

(b) $T=0.0625 \varepsilon / k$

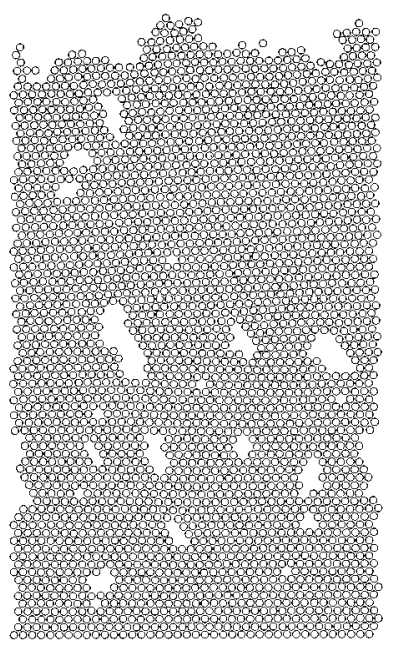

(c) $T=0.125 \varepsilon / k$

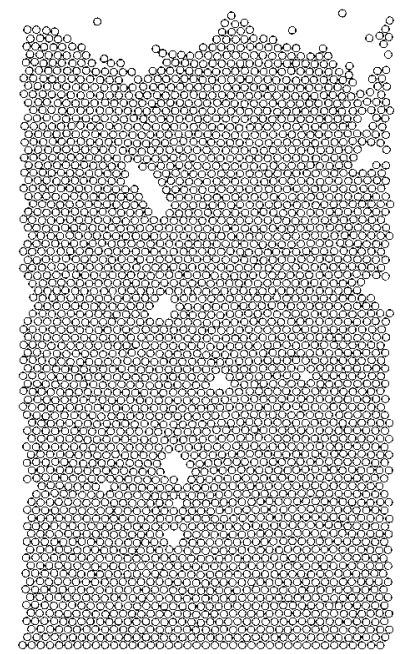

(d) $T=0.1875 \varepsilon / k$

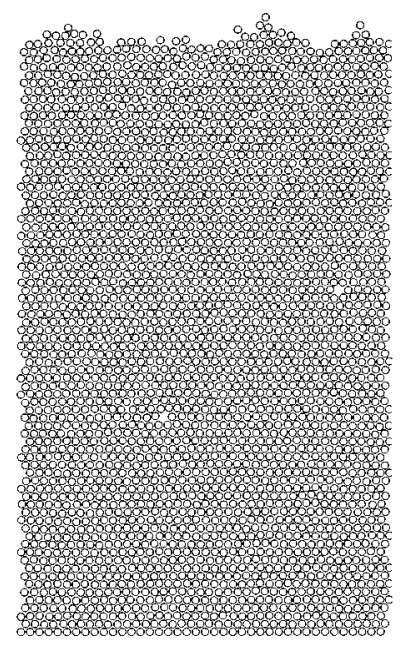

(e) $T=0.250 \varepsilon / k$

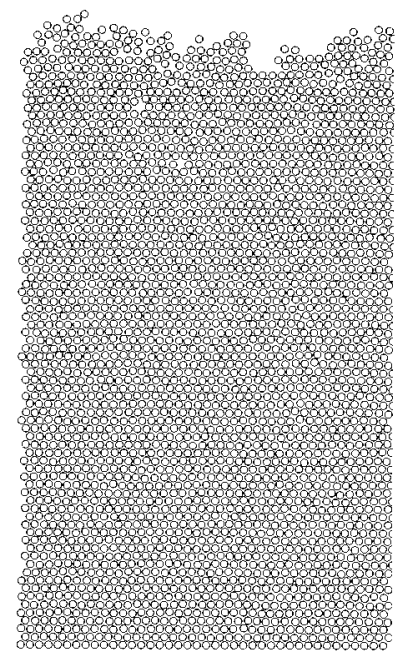

FIG. 2. Typical microstructures for films grown at various substrate temperatures under a deposition kinetic energy of $E_{b}=0.80 \epsilon$.

number of voids and the void volume fraction decrease with increasing temperature. While isolated vacancies are observed at all deposition temperatures, no voids are observed in the simulations at the two highest temperatures examined. At all temperatures where voids are observed, there is a tendency for the voids to be elongated in the direction parallel to the deposition flux (i.e., vertically). However, this is somewhat mitigated by the symmetry of the underlying crystal lattice. The voids tend to form in tracks parallel to the deposition direction (i.e., vertically), as may be seen most clearly in Fig. 2(c). The horizontal spacing between void tracks tends to increase with increasing temperature. The absence of voids in the films grown at the highest temperatures is likely attributable to the large void track spacing at high temperature and the small, finite width of the simulation cell.
Examination of Fig. 2 also shows that the roughness of the growth front (top surface) decreases with increasing temperature.

Several of the qualitative observations of the evolution of the film structure with substrate temperature are made more quantitative in Fig. 3. Figure 3(a) shows the evolution of the volume fraction of voids (including vacancies-single site voids), each averaged over three independent simulations. The decay of the void volume fraction with temperature is monotonic. As discussed above, the very small void volume fractions at the highest two temperatures may be somewhat lower than is physically reasonable owing to the increased void track spacing with increasing temperature and the small, finite width of the simulation cell. Unfortunately, we were unable to obtain adequate statistics on the variation 
(a)

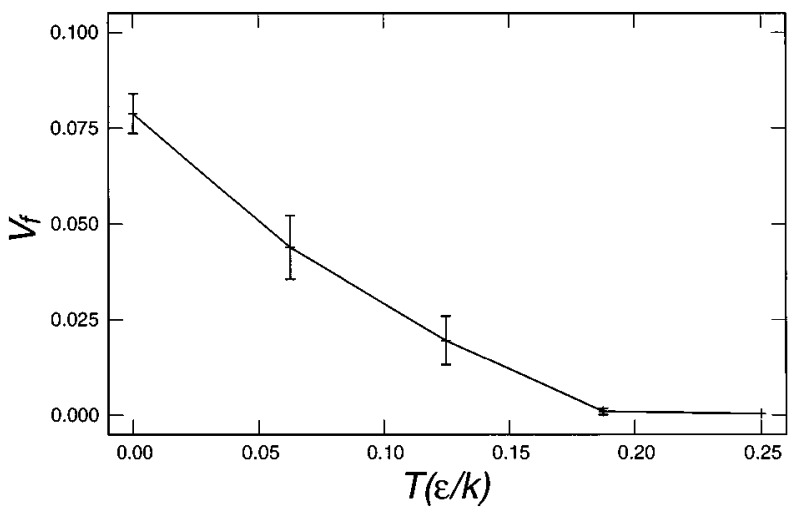

(b)

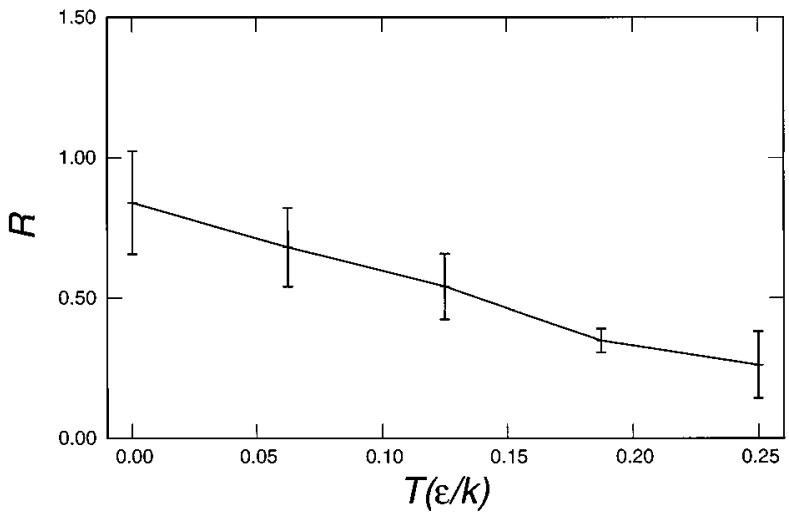

FIG. 3. Substrate temperature dependence of (a) void volume fraction, $V_{f}$, and (b) mean surface roughness, $R$, for films deposited at $E_{b}=0.80 \epsilon$. Error bars on void volume data reflect $\pm 1 \sigma$ for three independent simulations at each temperature. Surface roughness results reflect an average of ten measurements per simulation (spaced equally in time) for three independent simulations per temperature. Only one simulation was performed at $T$ $=0.25 \mathrm{\epsilon} / \mathrm{k}$.

of void track spacing with temperature to be more quantitative. The evolution of surface roughness $R$ (as defined above) with temperature is shown in Fig. 3(b), based upon measurements made at ten equally spaced time increments during the deposition of three independent simulations. Like the void volume fraction, the mean surface roughness decays monotonically with increasing temperature. However, the mean surface roughness decays more slowly than does the void volume fraction.

Microstructures of films grown at deposition kinetic energies $E_{b}=0.10,0.45,0.80,1.15,1.50$, and $3.00 \mathrm{\epsilon} /$ atom with a constant substrate temperature of $T=0.125 \epsilon / k_{B}$ are shown in Fig. 4. The dependence of the film microstructures on deposition kinetic energy shows a striking resemblance to those seen in Fig. 2. Namely, (i) no defects are observed at these values of $E_{b}$ other than voids and vacancies, (ii) the voids are elongated in the direction parallel to the deposition flux, (iii) the voids occur in vertical void tracks, the distance between void tracks increases with increasing deposition kinetic energy, and (iv) the void density decreases with increasing deposition kinetic energy. The similarity between the variation in microstructure with temperature and deposi- tion kinetic energy suggests that these two physical parameters play a similar role in the deposition process. The tendency of the voids to form in vertical tracks is especially apparent in the low energy sample. Both the number of voids and the void volume fraction decrease as the kinetic energy of the impinging particles is increased [Fig. 5(a)]. The mean surface roughness of the films is also found to be a decreasing function of incident kinetic energy as shown in Fig. 5(b).

All of the films grown under the range of deposition conditions described above were crystalline and maintained the crystallographic orientation of the substrate. In no cases were well-defined new grains formed. Nonetheless, occasionally dislocations were found in the lattice which locally rotated the crystal structure. Close examination of the atomic structure of the films, e.g., by sighting along the close packed direction (see Fig. 6), reveals that the vast majority of the dislocations lie within the voids. The presence of the dislocations causes a stress couple to be set up such that material surrounding part of the void is in compression and material on the other side of the void is placed in tension. This may be clearly seen in Fig. 6, where the spatial variation in the hydrostatic component of the local stress tensor around several voids is shown. The arrangement of atoms around the voids show that the Burgers vectors are of the magnitude and direction of nearest-neighbor vectors in the triangular lattice. Examples of dislocations with Burgers vectors corresponding to several of the allowed $60^{\circ}$ orientations may also be seen in Fig. 6 by consideration of the orientation of the compressive and tensile stress couples around individual voids. While many of the voids in Fig. 6 contain dislocations, many examples may also be found in which the voids are dislocation free. This suggests that voids do not nucleate on dislocations, rather that dislocations form on existing or incipient voids, as discussed below.

\section{DISCUSSION}

During the course of the film growth simulations, we recorded a sufficient number of images of the atomic structure to animate the growth process. Examination of these MD movies provided a means by which we could identify the void formation mechanism. This void formation process proceeds as follows: (i) the surface develops some roughness of a finite amplitude, (ii) deposited atoms falling into a depression in the film surface associated with this roughness are attracted to the walls of the depression due to the attractive part of the interatomic potential [Fig. 7(a)], (iii) the atoms deposited along the walls attract additional deposited atoms and begin to form a bridge across the depression [Fig. 7(b)], (iv) continued deposition completes the bridge, forming a closed void beneath it [Fig. 7(c)]. Because the range of atomic interactions is relatively short $\left(2.1 r_{0}\right.$ in the present simulations), void formation and the lateral growth of the bridge spanning a surface depression is essentially an atomic scale process. Earlier simulation studies that focused on the filling of trenches during the formation of integrated circuits $^{25}$ have shown the outward growth of trench sidewalls by essentially the same mechanism as described here. In that case, the tendency to form voids during the filling of trenches decreases with decreasing trench sidewall spacing, decreased 
(a) $E_{b}=0.10 \varepsilon$

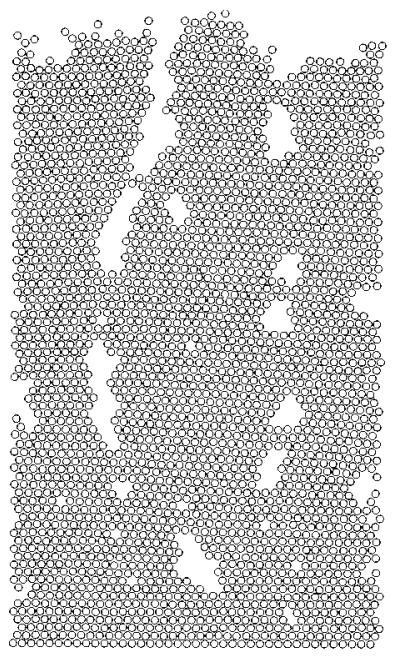

(b) $E_{b}=0.45 \varepsilon$

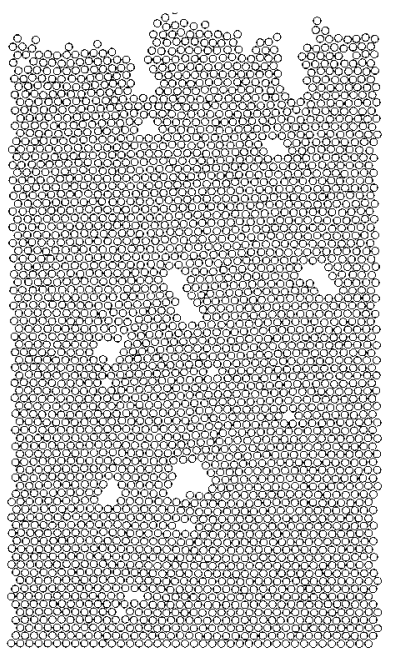

(c) $E_{b}=0.80 \varepsilon$

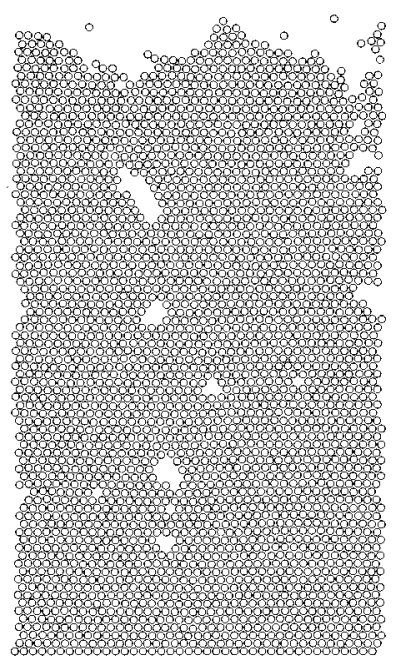

(d) $E_{b}=1.15 \varepsilon$

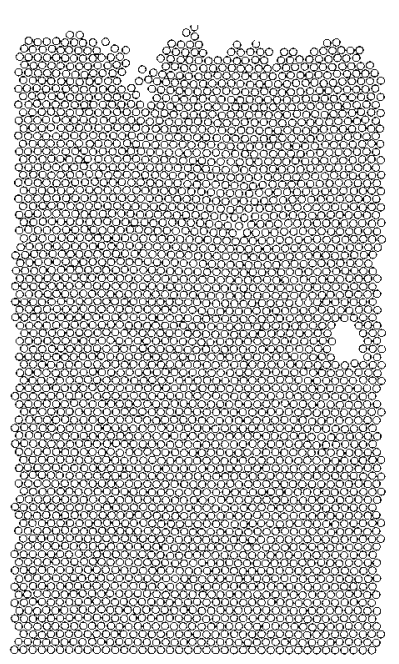

(e) $E_{b}=1.50 \varepsilon$

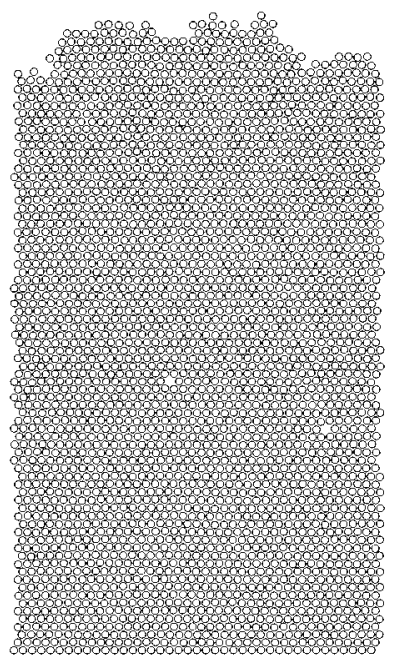

FIG. 4. Typical microstructures for films grown at various deposition kinetic energies onto a substrate at temperature $T=0.125 \epsilon / k$.

trench depth, and increased mobility of atoms on the surface. In the present study, the geometrical parameters describing the surface depression (or natural trench) are chosen by the growth process itself.

The growth of the bridges from the edges of the surface depression that lead to void formation generally occurs beneath the average film surface. Therefore, when the bridges pinch-off a new void [Fig. 7(c)], a surface depression above the new void remains (albeit much more shallow than prior to pinch-off). As the film continues to grow, this depression deepens (relative to the advancing average film surface). As a result, a new void will likely be formed by the same bridging process immediately above a previously formed void. This process is responsible for the vertical void tracks seen in Figs. 2 and 4. The correlation between a closed void, the associated surface depression above it, and the formation of a new void may be seen in Fig. 8 for a sample grown at $T=0.125 \epsilon / k$ and $E_{b}=0.80 \epsilon$.

As noted above, the observed mechanism for the formation of voids and void tracks requires the presence of surface depressions or surface roughness. This explains the fact that both the void volume fraction and the surface roughness show very similar dependencies on film growth conditions (i.e., substrate temperature and kinetic energy of the deposited atoms). However, while the surface roughness scales as the square of the product of the amplitude and wave number (inverse wavelength) of the surface roughness, the horizontal spacing between void tracks is expected to be associated only with the wavelength of the roughness. Therefore, we now reexamine the film roughness in terms of its separate components, wave number $(1 / \lambda)$, and amplitude $(A)$. Figure 9 shows the substrate temperature dependence of the wave 
(a)

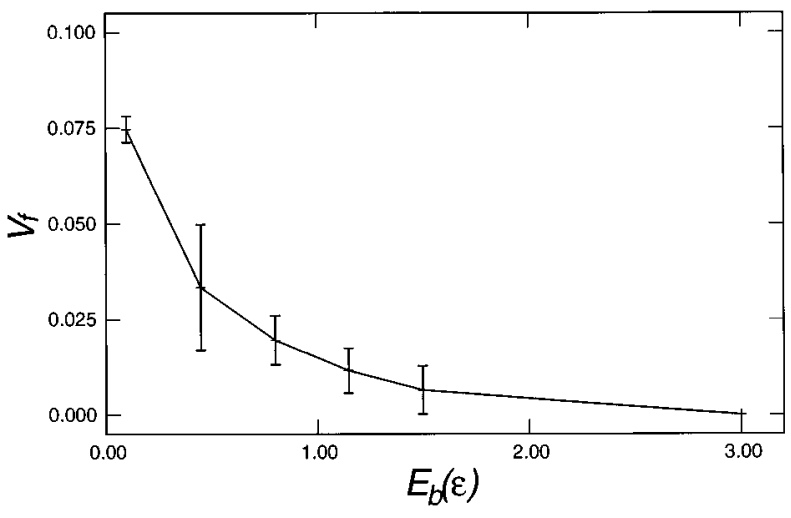

(b)

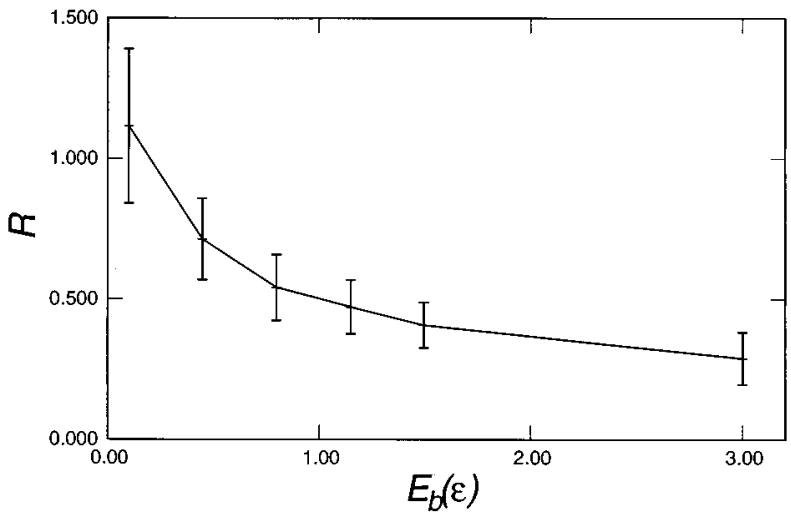

FIG. 5. Deposition kinetic energy dependence of (a) void volume fraction, $V_{f}$, and (b) mean surface roughness, $R$, for films deposited at $T=0.125 \mathrm{\epsilon} / \mathrm{k}$. Error bars on void volume data reflect $\pm 1 \sigma$ for three independent simulations at each energy. Surface roughness results reflect an average of ten measurements per simulation (spaced equally in time) for three independent simulations per energy. Only one simulation was performed at $E_{b}=3.0 \epsilon$.

number and amplitude of the surface roughness. Each point on the plot represents surface roughness data taken from ten equally spaced film thicknesses of each of three films. Like the overall roughness, both the wave number and amplitude of the surface roughness decrease monotonically with increasing temperature. A similar observation holds for the deposition kinetic energy dependence of the wave number and amplitude of the surface roughness (Fig. 10). As mentioned above, the horizontal spacing between void tracks increases with increasing temperature and deposition kinetic energy (although insufficient statistics are available to make this observation quantitative). The similarity between the void track spacing and the wavelength of the surface roughness provides further evidence of the surface origin of the void and void track formation mechanism.

Vertical void tracks are a common feature of low temperature film growth in metals and ceramics. Recently, these types of void tracks have also been observed in Si thin films grown via molecular beam epitaxy. ${ }^{18,19}$ In this work, the void tracks were found to be correlated to the presence of peaks and valleys in the surface formed as the result of the breakdown of the $\{100\}$ surface planes into $\{111\}$ facets. Although the surface roughness associated with faceting differs from

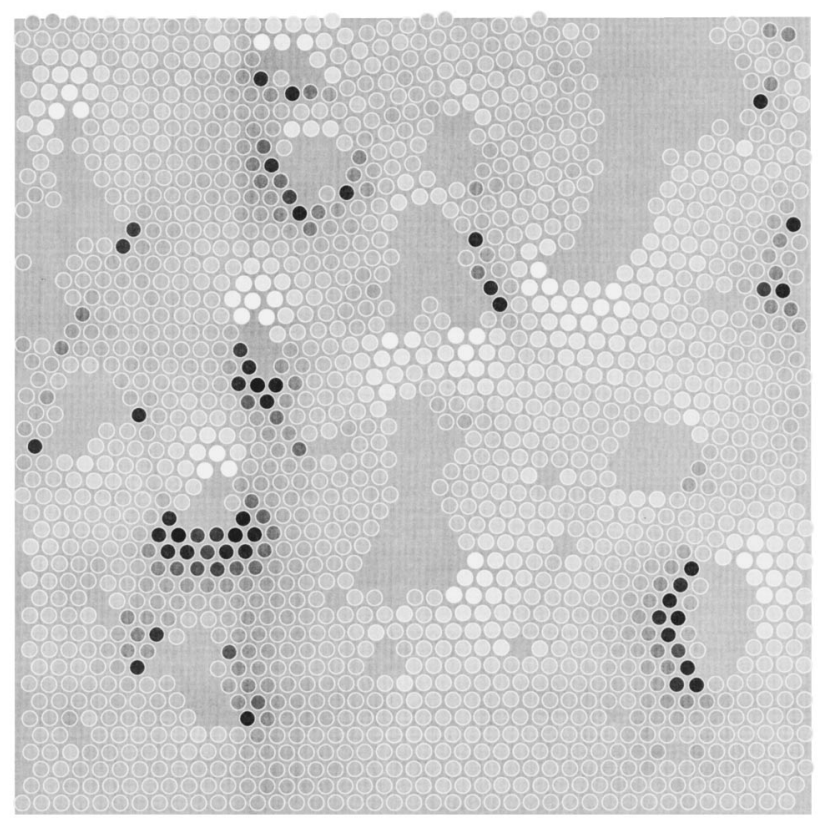

FIG. 6. Typical hydrostatic stress distribution around voids and dislocations formed during thin film growth. Dark shading indicates compressive stress, light tensile. The background gray level indicates zero hydrostatic stress. Maximum stresses in this example are within a factor of 2 of the theoretical strength of the material.

the type of surface roughness observed in the present simulations, the basic mechanism of void formation likely remains the same. Further, based upon their experimental observations, the authors ${ }^{18}$ speculated that the voids that formed were originally deep, open grooves that decomposed into a series of smaller, more spherical voids as film growth proceeds due to the Rayleigh instability. The present simulations suggests that the decomposition of the deep grooves into voids is a result of shadowing leading to the formation of bridges which pinch-off the void.

Comparisons of Figs. 3, 5, 9, and 10 reveal a strong similarity between the dependence of void volume fraction and the characteristic surface roughness on both the substrate temperature and deposition kinetic energy. This suggests that the deposition kinetic energy and the substrate temperature are modifying the physical process that controls void formation in the same way. As described above, the void formation mechanism is closely tied to the evolution of surface roughness. The reduction in surface roughness and void volume fraction with increasing temperature results from the enhanced diffusivity of atoms on the film surface. The MD movies of the deposition process clearly show that the enhanced atomic mobility on the surface increases the rate at which depressions in the film surface are filled and hills leveled. Since the formation of voids requires the formation of sufficiently deep surface depressions, any mechanism that levels the film surface will decrease the occurrence of sites sufficiently deep for the bridge development that leads to void formation. Just as increasing the temperature leads to increased atomic mobility on the surface, increases in the kinetic energy of the impinging atoms also increase the mobility of surface atoms. However, in the present simulations where the atomic diffusion distances are relatively small, the 


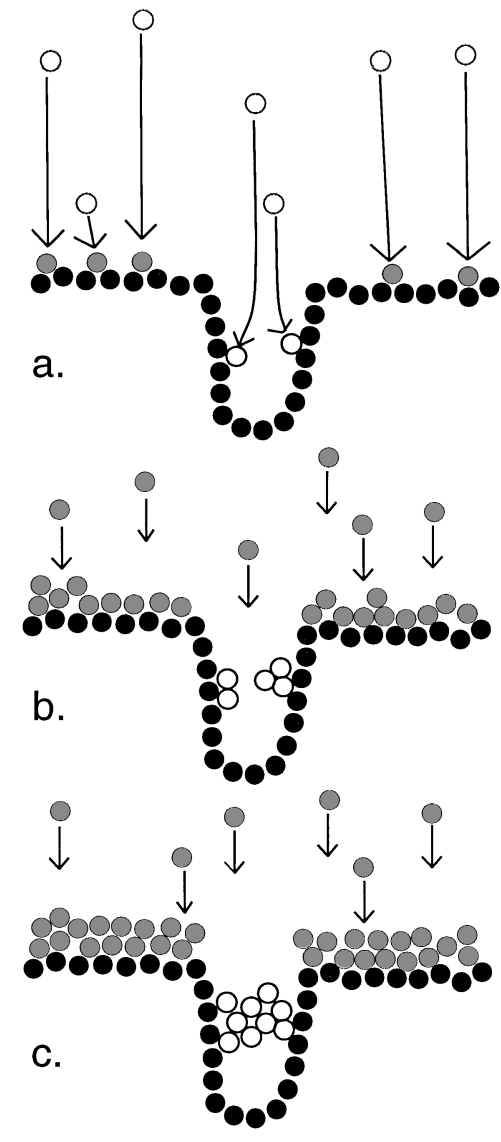

FIG. 7. Schematic representation of near surface void formation mechanism: (a) beam atoms are attracted to wall surfaces to form the nucleus of a bridge; (b) additional atoms begin to complete the bridge; (c) continued deposition forms a closed void with related surface depression above it.

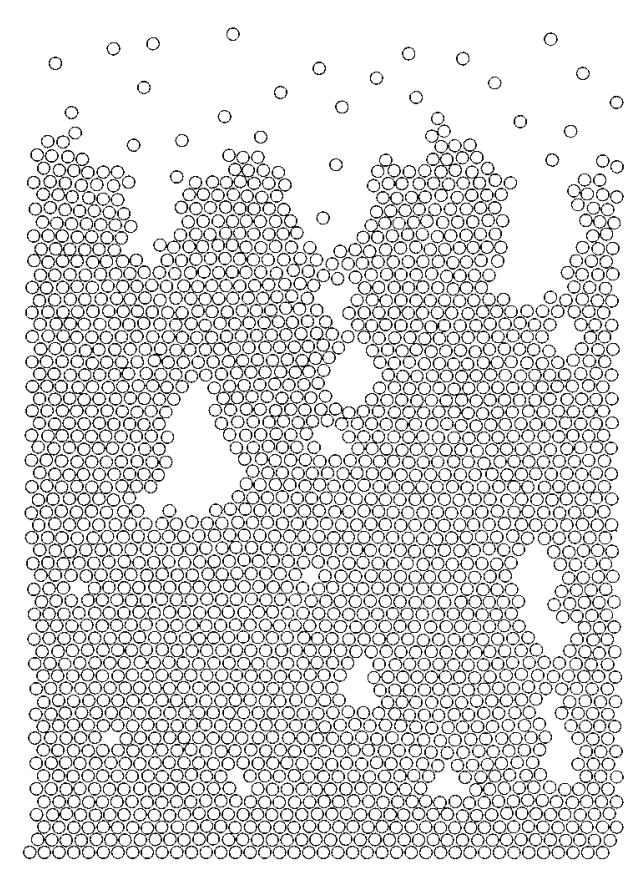

FIG. 8. Snapshot of film during growth at $T=0.125 \epsilon / k$ and $E_{b}=0.10 \epsilon$. The void track and correlation between voids and surface depressions are apparent. (a)

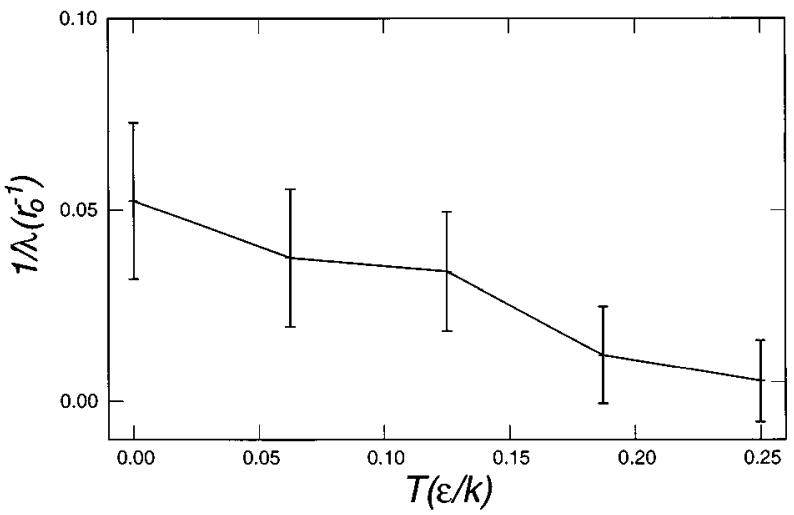

(b)

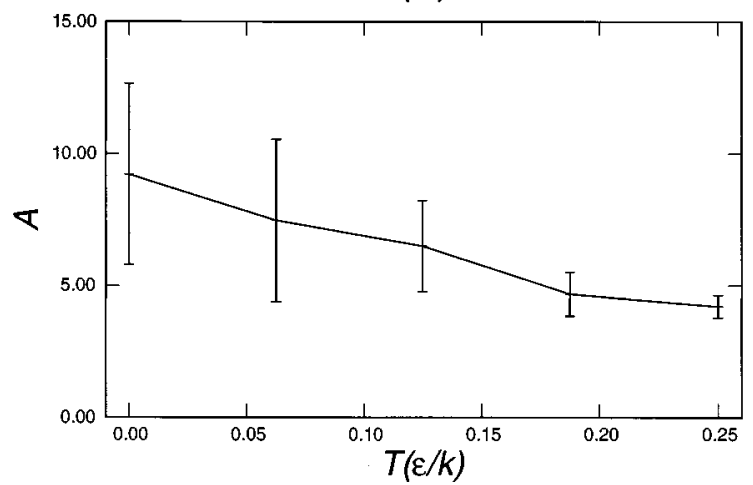

FIG. 9. Substrate temperature dependence of (a) wave number, $1 / \lambda$, and (b) amplitude, $A$, of surface roughness. Results reflect an average of ten measurements per simulation (spaced equally in time) for three independent simulations per temperature. Only one simulation was performed at $T$ $=0.25 \mathrm{\epsilon} / \mathrm{k}$.

predominant mechanism for the deposited atoms to increase the surface atom mobility is through kinematic interactions of the atoms in the vicinity of the deposition event. Although the motion of the atoms near the site of the impact are not behaving sufficiently incoherently to label this type of motion as heat, the impacts do lead to very localized increases in mobility. Therefore, increasing deposition energy and substrate temperature both serve as methods for increasing the surface atomic mobility, which decreases surface roughness, and thereby decreases the rate of void formation. For very large deposition kinetic energies, the impact of the deposited atoms is seen to disrupt the formation of hills on the surface, thereby reducing surface roughness. A similar effect has been proposed in the description of island dissociation during ion assisted deposition. ${ }^{26}$ This effect does not play a major role in modifying the surface roughness for the range of deposition kinetic energies where the void volume fraction is changing rapidly.

As described above in relation to Fig. 6, dislocations commonly form in thin films containing voids and these dislocations usually lie within the voids. These dislocations are all of edge character (screws cannot form in two-dimensional lattices) and are oriented such that the extra half-line (halfplane in three dimensions) of atoms lies either below the void or on the sides of the void and almost never above the 
(a)

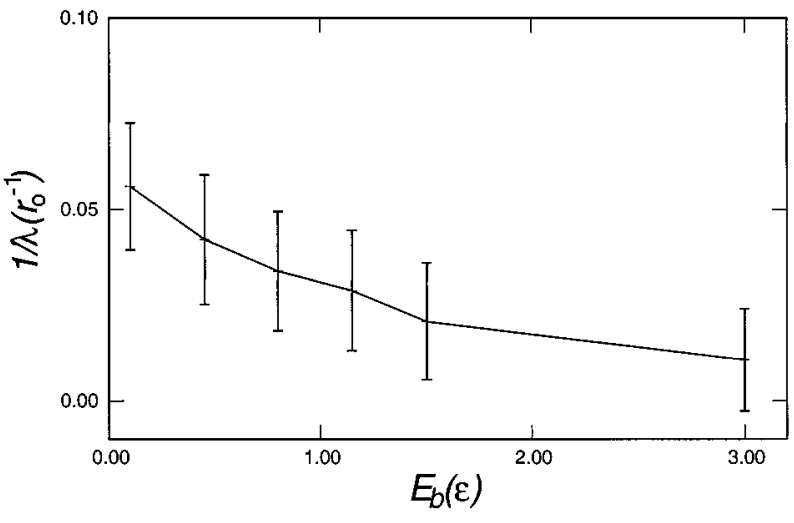

(b)

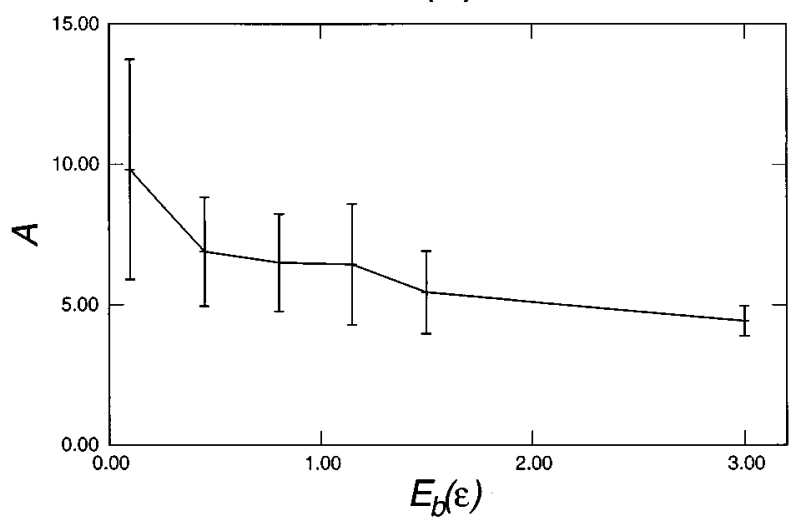

FIG. 10. Deposition kinetic energy dependence of (a) wave number, $1 / \lambda$, and (b) amplitude, $A$, of surface roughness. Results reflect an average of ten measurements per simulation (spaced equally in time) for three independent simulations per energy. Only one simulation was performed at $E_{b}=3.0 \epsilon$.

void. The orientation of these dislocation Burgers vectors is a direct result of the mechanism by which the dislocations form. As a bridge across a void begins to form from the outgrowth of atoms from the sides of a surface depression, it eventually reaches a configuration such that the outgrowths from the two sidewalls are within a single atomic spacing of each other. In this configuration, the atoms at the ends of the two outgrowths attract each other through the interatomic potential. If the stiffness of the material in the outgrowths and/or sidewalls is not too large, these two outgrowths joinleading to a tensile stress in the resulting bridge and a missing half-line above the void. Additional atoms deposited on top of this bridge form a slightly distorted crystal that is commensurate with this missing half-line bridge. This is the mechanism by which dislocations are formed in voids. The more heavily voided the film is, the lower the effective modulus of the material in the void vicinity and the greater the propensity for dislocation formation within the voids. Depending on the orientation of the bridge, dislocations can also form with Burgers vectors that are not parallel with the surface.

In summary, the MD method has been used to simulate the growth of thin films, deposited at a variety of substrate temperatures and deposition kinetic energies. The principal microstructural features to develop within these films are single vacancies and small voids which tend to be slightly elongated and to be aligned in the growth direction. Both the void volume and the mean surface roughness of the films are found to be decreasing functions of substrate temperature and deposition kinetic energy. Voids are shown to form as a consequence of both surface roughness and shadowing effects. The attraction between deposited atoms and the sides of surface depressions lead to the formation of outgrowths from the sidewalls of the surface depression. These outgrowths shadow the open void beneath them and continue to grow across the voids by interaction with the depositing atoms until a continuous bridge is formed that closes off the void. Since this bridging mechanism leaves behind a surface depression above the closed-off void, new voids tend to form above them. This leads to the alignment of voids along the film growth direction. The spacing of the resultant void tracks is correlated with the wavelength of the surface roughness. Increasing temperature and deposition kinetic energy enhancing surface mobility leads to an increase in the wavelength of the surface roughness and hence an increase in the spacing between void tracks. Edge dislocations tend to form within voids as a natural consequence of the void bridging process, however nondislocated voids are also observed.

\section{ACKNOWLEDGMENTS}

This work was supported by the Advanced Projects Research Agency and the Office of Naval Research under Contract No. N00014-91-J-4019. The authors wish to thank Professor Steve Yalisove, Professor Brad Orr, and Dr. Jerry Tersoff for useful discussions during the performance of this work.

${ }^{1}$ B. A. Movchan and A. V. Demchishin, Phys. Met. Metalogr. 28, 83 (1969).

${ }^{2}$ J. A. Thornton, Annu. Rev. Mater. Sci. 7, 239 (1977).

${ }^{3}$ D. Liu, S. K. Dew, M. J. Brett, T. Janacek, T. Smy, and W. Tsai, J. Appl. Phys. 74, 1339 (1993).

${ }^{4}$ A. G. Dirks and H. J. Leamy, Thin Solid Films 47, 219 (1977).

${ }^{5}$ P. Smilauer, M. R. Wilby, and D. D. Vvedensky, Phys. Rev. B 47, 4119 (1993).

${ }^{6}$ P. Smilauer, M. R. Wilby, and D. D. Vvedensky, Phys. Rev. B 48, 4968 (1993).

${ }^{7}$ J. Salik, J. Appl. Phys. 57, 5017 (1985).

${ }^{8}$ Z. Zhang, J. Detch, and H. Metiu, Phys. Rev. B 48, 4972 (1993).

${ }^{9}$ K.-H. Müller, J. Appl. Phys. 58, 2573 (1985).

${ }^{10}$ Y. Sasajima, H. Tanaka, M. Ichimura, M. Itaba, and S. Ozawa, Jpn. J. Appl. Phys. 32, 2037 (1993).

${ }^{11}$ S. Müller-Pfeiffer, H. Van Kranenburg, and J. C. Lodder, Thin Solid Films 213, 143 (1992).

${ }^{12}$ P. Stoltze and J. K. Nørskov, Phys. Rev. B 48, 5607 (1993).

${ }^{13}$ K.-H. Müller, Phys. Rev. B 35, 7906 (1987).

${ }^{14}$ S. M. Paik, S. Kim, I. K. Schuller, and R. Ramirez, Phys. Rev. B 43, 1843 (1991).

${ }^{15}$ K.-H. Müller, J. Appl. Phys. 62, 1796 (1987).

${ }^{16}$ S. J. Cook and P. Clancy, J. Chem. Phys. 99, 2175 (1993).

${ }^{17}$ G. H. Gilmer, M. H. Grabow, and A. F. Bakker, Mater. Sci. Eng. B 6, 101 (1990).

${ }^{18}$ D. D. Perovic, G. C. Weatherly, J.-P. Noël, and D. C. Houghton, J. Vac. Sci. Technol. B 9, 2034 (1991).

${ }^{19}$ D. D. Perovic, G. C. Weatherly, P. J. Simpson, P. J. Schultz, T. E. Jackman, G. C. Aers, J.-P. Noël, and D. C. Houghton, Phys. Rev. B 43, 14257 (1991).

${ }^{20}$ D. P. Adams, M. Vill, J. Tao, J. C. Bilello, and S. M. Yalisove, J. Appl. Phys. 74, 1015 (1993). 
${ }^{21}$ B. J. Adler and T. E. Wainwright, J. Chem. Phys. 31, 459 (1959).

${ }^{22}$ J. R. Beeler, Jr., Radiation Effects Computer Experiments (North-Holland, New York, 1983).

${ }^{23}$ M. P. Allen, D. J. Tildesley, Computer Simulation of Liquids (Clarendon, Oxford, 1987).
${ }^{24}$ S.-P. Chen, T. Egami, V. Vitek, Phys. Rev. B 37, 2440 (1988).

${ }^{25}$ D. S. Band, J. P. McVittie, K. C. Saraswat, J. A. Iacoponi, J. Gray, Z. Krivokapic, and K. A. Littau, Mater. Res. Soc. Symp. Proc. (in press).

${ }^{26}$ J. M. Millunchick, L. Hultman, and S. A. Barnett, J. Vac. Sci. Technol. A 13, 1155 (1995). 